\title{
In Vitro and In Vivo Efficacy of DNA Damage Repair Inhibitor Veliparib in Combination with Artesunate against Echinococcus granulosus
}

\author{
Y. F. Li $\mathbb{D}^{1,2,3,4}$ L. M. Wen ${ }^{10},{ }^{1,2,3}$ J. Zhao, ${ }^{1,3}$ G. D. Lv, ${ }^{3}$ S. Lu $\mathbb{D}^{5},{ }^{5}$ S. Lu, ${ }^{1,3}$ X. Zheng, ${ }^{2}$ B. Chen, ${ }^{1,3}$ \\ C. Y. Tian, ${ }^{2}$ Y. H. Gong, ${ }^{1,3}$ H. J. Gao, ${ }^{1,3}$ and J. H. Wang $\oplus^{1,3}$ \\ ${ }^{1}$ Pharmaceutical Department, The First Affiliated Hospital of Xinjiang Medical University, Urumqi 830054, China \\ ${ }^{2}$ College of Pharmaceutical Sciences, Xinjiang Medical University, Urumqi 830054, China \\ ${ }^{3}$ State Key Laboratory of Pathogenesis, Prevention, Treatment of Central Asian High Incidence Diseases, Urumqi 830054, China \\ ${ }^{4}$ Clinical Pharmacy, The Third Affiliated Hospital of Bengbu Medical College, Suzhou 234000, China \\ ${ }^{5}$ Department of Orthopedics, The First Affiliated Hospital of Xinjiang Medical University, Urumqi 830054, China
}

Correspondence should be addressed to Y. F. Li; efiafen1037@163.com, L. M. Wen; wendywenlimei@163.com, and J. H. Wang; jhw716@sohu.com

Received 27 June 2019; Revised 30 September 2019; Accepted 17 October 2019; Published 4 July 2020

Academic Editor: Massimiliano M. Corsi Romanelli

Copyright (c) 2020 Y. F. Li et al. This is an open access article distributed under the Creative Commons Attribution License, which permits unrestricted use, distribution, and reproduction in any medium, provided the original work is properly cited.

Cystic echinococcosis (CE), caused by the cestode Echinococcus granulosus, is a worldwide chronic zoonosis. Albendazole (ABZ) and mebendazole are effective against $\mathrm{CE}$, but a high dosage in a long-term period is usually required. In this study, we evaluate the effects of DNA damage repair inhibitor (i.e., Veliparib) in combination with artesunate (AS) on hydatid cysts. For the in vitro assay, protoscoleces of E. granulosus (E.g PSCs) were incubated with low AS (AS-L, $65 \mu \mathrm{M}$ ), moderate AS (AS-M, $130 \mu \mathrm{M})$, and high AS (AS-H, $325 \mu \mathrm{M})$, AS-L/M/H+Veliparib $(10 \mu \mathrm{M})$, and ABZ $(25 \mu \mathrm{M})$, respectively. The AS-H+Veliparib group showed the maximal protoscolicidal effects. Ultrastructural change revealed that germinal layer (GL) cells were reduced, and lipid droplets appeared. AS could induce DNA injuries in PSCs. The 8-OHdG was expressed in the PSCs and GL of the cysts in mice, especially in the presence of Veliparib. The most severe DNA damages were observed in the AS-H+Veliparib group. Meanwhile, the expression of ribosomal protein S9 (RPS9) gene in the AS-H+Veliparib group was significantly lower than that in the AS-H group. The in vivo chemotherapeutic effects of AS-L ( $50 \mathrm{mg} / \mathrm{kg}), \mathrm{AS}-\mathrm{H}(200 \mathrm{mg} / \mathrm{kg})$, and AS-H+Veliparib $(25 \mathrm{mg} / \mathrm{kg})$ were assessed in experimentally infected mice. Upon 6 weeks of oral administration, ultrasonography was used to monitor the volume change of vesicles. Maximum potentiation was seen on day 15 with values (versus AS) of $34(P<0.05)$ for AS-H + Veliparib. It led to the reduction of cyst weight $(55.40 \%)$ compared with the model group $(P<0.01)$, which was better than AS alone (52.84\%) and ABZ-treated mice (55.35\%). Analysis of cysts collected from AS-H+Veliparib-treated mice by transmission electron microscopy revealed a drug-induced structural destruction. The structural integrity of the germinal layer was lost, and the majority of the microtriches disappeared. In conclusion, our study demonstrates that AS or AS in combination with Veliparib is effective for treating CE, especially the combination group. On this basis, AS represented promising drug candidates in anti-CE chemotherapy.

\section{Introduction}

Cystic echinococcosis (CE), also known as cystic hydatid disease, is a zoonotic infection caused by larval stage of Echinococcus granulosus $[1,2]$. Nowadays, benzimidazole compounds, particularly the albendazole (ABZ), have been reported to be effective against CE [3]. However, these compounds, with poor water solubility, were classified as type II drugs on the Biopharmaceutical Classification System [4]. The low dissolution rate of ABZ triggers insufficient absorp- 
tion in the gastrointestinal tract, resulting in a low plasma drug level [5]. Moreover, long-term medication of ABZ results in severe adverse events in these patients. Therefore, novel chemotherapeutic agents with good efficacy are urgently required.

Artemisinin, an ancient Chinese herbal, was initially isolated in 1972 from Artemisia annua. L. The World Health Organization (WHO) recommends the artemisinin-based combination therapy (ACT) as the first-line treatment for Plasmodium falciparum infection [6]. In recent years, artemisinin and its derivatives have been reported to show curative effects on protozoans [7], nematodes [8], trematodes, and tumor [9]. These findings prompted us to investigate the potency of artemisinin for treating CE. Artemisinin and its derivatives including artesunate (AS) showed high efficiency with low toxicities. The effects of AS were further evaluated using the BALB/c mouse model $[10,11]$. AS combined with $A B Z$ resulted in obvious reduction of the cyst weights compared to single application of AS [12]. However, the main mechanisms of AS against parasites are still not clear.

The modulatory effects of AS on the parasite transcriptome were mainly associated with the regulation of protein involved in encoding companion, transcriptional activation, proteasome, oxidative stress, and cell cycles [13]. Parasite exposure to AS resulted in a rapid depolarization of parasite $\Delta \Psi \mathrm{m}$ and $\Delta \Psi \mathrm{p}$, which was shown to be mediated via the generation of ROS initiated by iron bioactivation of endoperoxides and/or catalysed by irondependent oxidative stress [14]. Therefore, we speculated that the main pharmacological roles of artemisinin were associated with the generation of free radicals by the endoperoxide bridge and induction of oxidative stress, which ultimately led to parasitic death $[15,16]$. In our previous study, 15 genes associated with the genetic processing were upregulated after artemisinin treatment, including DNA replication, repair, transcription, and translation [17]. Meanwhile, Wen et al. [18] firstly cloned the RPS9 gene that participated in DNA repair and developmental regulations from the E. granulosus protoscoleces under oxidative stress induced by AS.

DNA repairing mechanism is usually activated upon DNA damages. Efficient DNA repair is an important mechanism for resistance. Therefore, inhibition of DNA repair would be the most effective for this process [19, 20]. The oxidative stress-induced DNA damages were mainly repaired by base excision repair (BER) [21] that involved the base pair deletion and generation of $8-\mathrm{OHdG}$ [22]. As an important component of the BER pathway, poly (ADP-ribose) polymerase (PARP) is a multifunctional protein involved in the activation of the BER downstream repairing protein recruiting the DNA polymerase and ligase. Besides, it closely mediated the DNA damage recognition and signal transmission. Among the PARP family, PARP-1 is the extensively studied [23]. In the early-stage clinical screening, Veliparib (ABT-888) was considered the third-generation inhibitor of PARP-1, with high activity and selectivity, exerting higher efficiency in combination with other agents [24].

In this study, we aim to investigate the efficiency of Veliparib combined with AS on treating CE. For the in vitro experiments, eosin staining and alkaline phosphatase activity assay were utilized to detect protoscoleces (PSCs) activity. The ultrastructural change of PSCs were observed by transmission electron microscopy (TEM). For the in vivo assay, ultrasonography was used to monitor the volume change of vesicles, and calculate the cyst inhibition rate. TEM was used to evaluate the efficiency of AS and the combination of AS and Veliparib on treating CE.

\section{Materials and Methods}

2.1. In Vitro Culture of PSCs. PSCs were obtained from hydatid cysts of naturally infected sheep livers that were $>$ collected from abattoirs in Urumqi, Xinjiang Uyghur Autonomous Region. PSCs were digested using 1\% of pepsin in distilled water at pH 2.0 for $30 \mathrm{~min}$. Cyst culture was performed under aseptic conditions according to our previous description [18].

2.2. In Vitro Drug Treatment of PSCs. PSCs were seeded in 96-well cell culture plates in $0.2 \mathrm{~mL}$ cultivation medium at $37^{\circ} \mathrm{C}$ with $5 \% \mathrm{CO}_{2}$. The PSCs were divided into the following groups: (a) DMSO group, treatment with $1 \%(v / v)$ DMSO; (b) Veliparib group, treatment with $10 \mu \mathrm{M}$ Veliparib; (c) ABZ group, treatment with $50 \mu \mathrm{M} \mathrm{ABZ}$, serving as a positive control; (iv) AS-L group, treatment with $65 \mu \mathrm{M}$ AS; (d) AS-M group, treatment with $130 \mu \mathrm{M}$ AS; (e) AS-H group, treatment with $325 \mu \mathrm{M}$ AS; (f) ABZ+Veliparib group, treatment with $50 \mu \mathrm{M} \mathrm{ABZ}$ and $10 \mu \mathrm{M}$ Veliparib; (g) AS-L + Veliparib group, treatment with $65 \mu \mathrm{M}$ AS and $10 \mu \mathrm{M}$ Veliparib; (h) AS-M + Veliparib group, treatment with $130 \mu \mathrm{M}$ AS and $10 \mu \mathrm{M}$ Veliparib; (i) and AS-H+Veliparib group, treatment with $325 \mu \mathrm{M}$ AS and $10 \mu \mathrm{M}$ Veliparib. In the AS combined with Veliparib groups, Veliparib was added about $30 \mathrm{~min}$ before AS. AS (B20992) and ABZ (A822534-50) were purchased from Yuanye Biotechnology (Shanghai, China). Veliparib (S1004c), purchased from Selleck Chemical (Shanghai, China), was dissolved in DMSO for subsequent analysis. After 4 days of drug treatment, PSCs were collected every day, and each experiment was performed at least in triplicate.

2.3. Eosin Staining and ALP Determination. PSCs viability was assessed by $1 \%$ Eosin staining followed by morphologic observation. The corresponding numbers of viable/nonviable PSCs were determined. We determined the activity of $E$. granulosus alkaline phosphatase (EgALP) in culture medium supernatants of PSCs. During the 4-day treatment, $50 \mu \mathrm{L}$ culture supernatant was collected daily and stored at $-80^{\circ} \mathrm{C}$ to measure EgALP activity [25] using the commercial alkaline phosphatase (ALP) Assay Kit (Beyotime Biotech, Shanghai, China). After drug treatment, the treated PSCs were collected, and TEM was used to observe the PSCs as described previously [26]. Micrographs were taken on a JEM-100CX II system.

2.4. Comet Assay. In the comet assay, PSCs were divided into the following groups: DMSO group, treatment with $1 \%$ DMSO; ABZ group, treatment with $50 \mu \mathrm{M}$ ABZ; Veliparib group, treatment with $10 \mu \mathrm{M}$ Veliparib; AS-L group, treatment with $65 \mu \mathrm{M}$ AS; AS-H group, treatment with $325 \mu \mathrm{M}$ 
AS; and AS-H+Veliparib group, treatment with $325 \mu \mathrm{M}$ AS and $10 \mu \mathrm{M}$ Veliparib. After culture for 4 days, the PSCs were collected for comet assay according to the method proposed by Singh et al. [27], with some minor modifications. The microscope slides were immerged with $0.6 \%$ agarose, followed by incubating at $65^{\circ} \mathrm{C}$ overnight. The PSCs culture solution was removed and then was washed using PBS. Then, centrifugation was performed; $90 \mu \mathrm{L}$ of $0.75 \%$ agarose was used to resuspend the PSCs onto the first layer of agarose. After agarose solidification at $4^{\circ} \mathrm{C}$ for $5 \mathrm{~min}$, the coverslips were removed and the slides were immersed for $4 \mathrm{~h}$ at $4^{\circ} \mathrm{C}$ in lysis solution freshly prepared $(2.5 \mathrm{M} \mathrm{NaCl}, 100 \mathrm{mM}$ $\mathrm{Na}_{2}$ EDTA, and $10 \mathrm{mM}$ Tris-HCl) containing $1 \%$ Triton X100 and $10 \%$ dimethyl sulfoxide. The slides were equilibrated in alkaline solution $\left(1 \mathrm{mM} \mathrm{Na} \mathrm{N}_{2} \mathrm{EDTA}, 300 \mathrm{mM} \mathrm{NaOH}\right.$, $\mathrm{pH} 13$ ) for $20 \mathrm{~min}$. Electrophoresis was carried out for $20 \mathrm{~min}$ at $25 \mathrm{~V}$ and $0.83 \mathrm{~V} / \mathrm{cm}$. After this, slides were neutralized by washing with $0.4 \mathrm{M}$ Tris- $\mathrm{HCl}$ buffer $(\mathrm{pH} 7.5)$ for $15 \mathrm{~min}$. Slides were stained with $60 \mu \mathrm{L}$ propidium iodide solution in the dark.

The images were observed under a magnification of 200x using a fluorescent microscope (Olympus IX73, Japan), and the image for PSCs was acquired using the CASP software. Finally, PSCs were analyzed using the percentage tail DNA of comets [28].

2.5. Determination of DNA Damages in E.g PSCs. Immunodetection of 8-OHdG adducts conducted as previously described [29] with some modifications. The DMSO (1\%, $v / v)$, ABZ $(50 \mu \mathrm{M})$, Veliparib $(10 \mu \mathrm{M})$, AS-L/H $(65 \mu \mathrm{M}$, $325 \mu \mathrm{M}$ ), and AS-H+Veliparib-treated PSCs for 4 days were fixed in $4 \%$ paraformaldehyde for $24 \mathrm{~h}$ at $4{ }^{\circ} \mathrm{C}$. After dehydration, sections were incubated at room temperature for 10 min in $3 \% \mathrm{H}_{2} \mathrm{O}_{2}$ and washed three times in PBS. Slides were placed in a container with citrate buffer, followed by heating in a microwave oven at about $98^{\circ} \mathrm{C}$. After cooling at room temperature, the slides were blocked with normal goat serum at $37^{\circ} \mathrm{C}$ for $20 \mathrm{~min}$ and incubated with monoclonal mouse antibodies anti 8-OHdG $(1: 100$, no. ab62623, Abcam, USA) overnight at $4^{\circ} \mathrm{C}$. Subsequently, the mixture was incubated with the secondary goat anti-mouse FITC antibody (1:400, no. ab6785, Abcam, USA). Sections were washed three times and with Hoechst 33258 staining. Afterwards, slides were mounted in visualized in a fluorescent microscope (Olympus IX73, Japan), and then, the images of nuclei presenting DNA damage were obtained.

2.6. Quantitative Real-Time PCR. PSCs were exposed to cultivation medium, ABZ $(50 \mu \mathrm{M})$, Veliparib $(10 \mu \mathrm{M})$, AS-L/H (AS-L, $65 \mu \mathrm{M}$; AS-H, $325 \mu \mathrm{M}$ ), and AS-H+Veliparib. After 4 days of drug treatment, total RNA was extracted from freshly isolated PSCs using Trizol reagent (Invitrogen, USA); cDNA was synthesized according to our previous description [17]. For the identification of EgRPS9 mRNA expression, real-time PCR reaction was carried out as previously described [17].

2.7. Western Blot Analysis. Proteins extracted from PSCs were subjected to SDS-PAGE. Separated proteins were elec- trophoretically transferred to polyvinylidene difluoride (PVDF) membranes (Merck Millipore, Darmstadt, Germany) and immunoblotted with a rabbit anti-RPS9 antibody (ab74711, Abcam, Cambridge, UK). A rabbit anti- $\beta$-actin antibody (ab205718, Abcam) served as the loading control. Immunoreactive proteins were visualized using the Odyssey Infrared Imaging System (Li-Cor, Lincoln, Nebraska) as described by the manufacturer. The relative intensities of the bands were quantified by densitometric analysis. The densitometric plots of the results were normalized to the intensity of the $\beta$-actin band.

2.8. In Vivo Treatment with Compounds. The animal handling was conducted with the approval of the Institutional Animal Care and Use Committee, the First Affiliated Hospital of Xinjiang Medical University (No. IACUC-2015022570). Pathogen-free female Kunming mice (6-8 weeks) were purchased from Xinjiang Medical University Animal Experiment Center and raised in the animal facility of the First Affiliated Hospital of Xinjiang Medical University.

For the infection challenge of mice, PSCs were precultured in vitro to generate small cysts (microcysts, 200$300 \mu \mathrm{m}$ in diameter) [30]. Each mouse was intraperitoneally transplanted with 25 microcysts suspended in $0.3 \mathrm{~mL}$ RPMI 1640 medium. About 180 days postinfection, the mice were randomly divided into seven groups including (a) the control group, normal mice with no infection challenge $(n=10)$; (b) model group, PSC-infected mice that received $500 \mu \mathrm{L}$ of $0.4 \%$ CMC-Na via lavage; (c) Veliparib group, PSC-infected mice that received Veliparib $(25 \mathrm{mg} / \mathrm{kg})$ dissolved in $0.9 \% \mathrm{NaCl}$ solution ( $\mathrm{pH} 4.0$ ); (d) ABZ group, PSC-infected mice that received $A B Z$ with a concentration of $200 \mathrm{mg} / \mathrm{kg}$ via lavage; (e) AS-L group, PSC-infected mice that received $50 \mathrm{mg} / \mathrm{kg}$ AS via lavage; (f) AS-H group, PSC-infected mice received $200 \mathrm{mg} / \mathrm{kg}$ AS via lavage; and (g) AS-H+Veliparib group, PSC-infected mice that received Veliparib $(25 \mathrm{mg} / \mathrm{kg})$ combined with $200 \mathrm{mg} / \mathrm{kg}$ AS. The lavage of AS-H group lasted for 6 weeks. The size of the cysts in mice was determined using ultrasonography according to the formula of $\left(\right.$ length $\times$ width $\left.^{2}\right) / 2$. The volume growth rate of cysts was calculated as follows: $\mathrm{T} / \mathrm{C} \%=$ (mean cyst volume in the test group/mean cyst volume in the control group) $\times 100 \%$.

The cysts were separated after treatment, followed by weighing the cyst. Then the wet weight of cysts and cystgrowth inhibiting rate were calculated using the following formula: cyst growth inhibiting rate $=$ (wet weight of cyst in the control - wet weight of cyst in the treatment group)/wet weight of cyst in the model control $\times 100 \%$.

2.9. TEM. For probing tissue changes after drug treatments, cysts were processed for TEM analysis as described previously [26]. Micrographs were taken on a JEM-100CX II TEM.

2.10. Statistical Analysis. All data were shown as the mean \pm standard deviation. SPSS 19.0 software was utilized for the data analysis. The univariate analysis of variance was used to compare the viability rate of PSCs between the experimental groups in vitro. Student's $t$-test and chi 


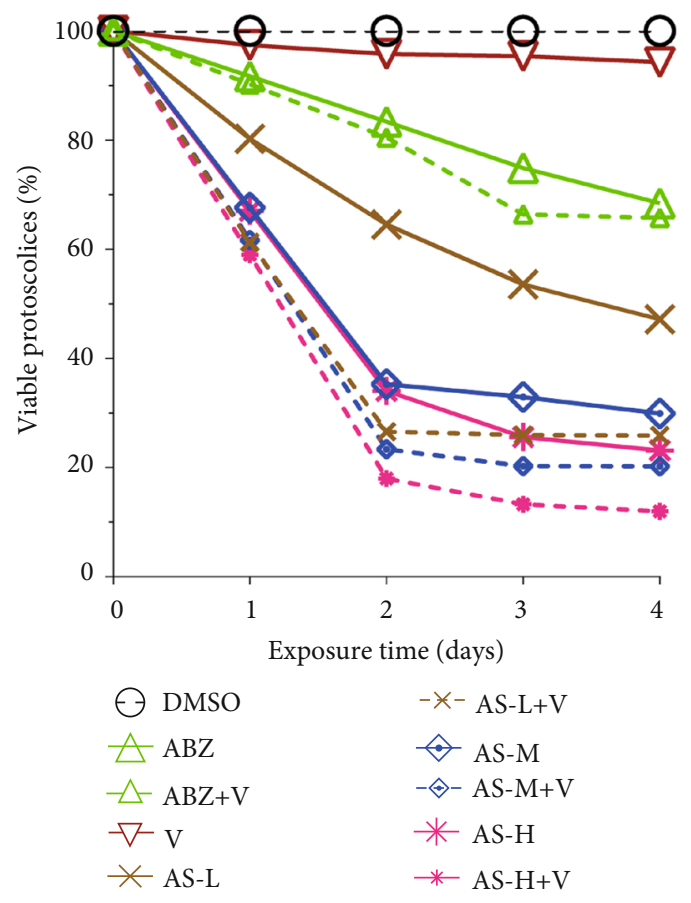

(a)

DMSO

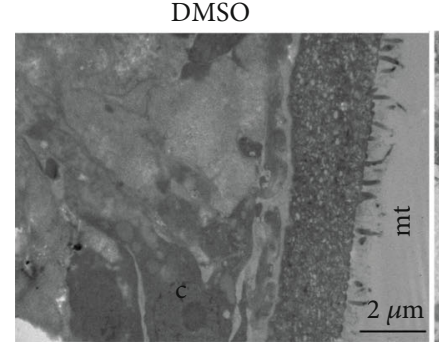

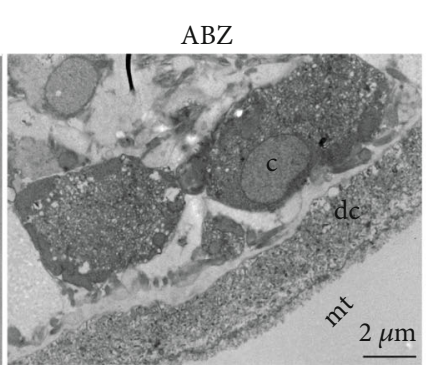
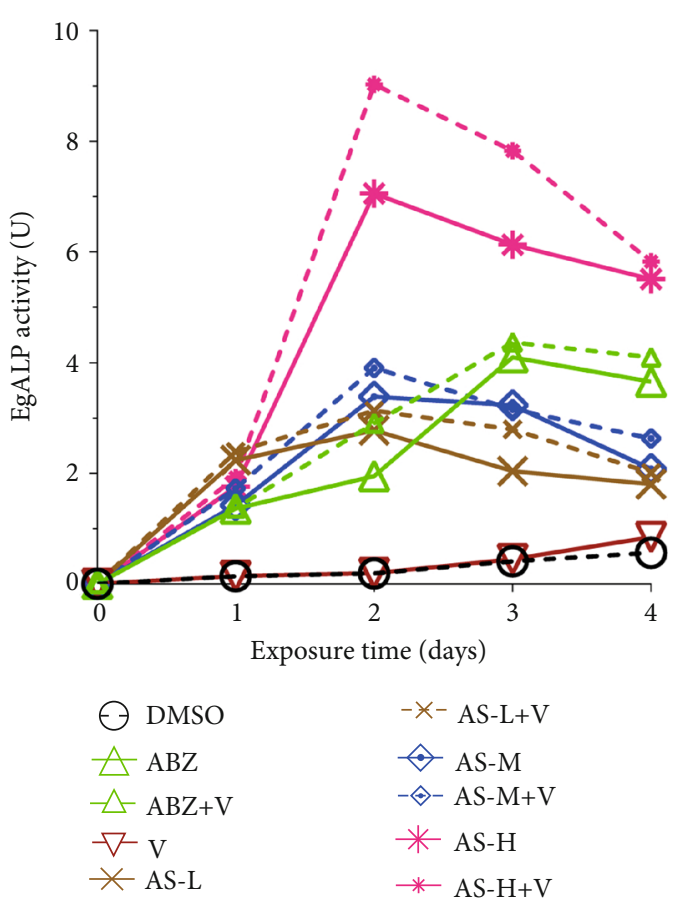

$-*-$ AS-L+V
$\odot$ AS-M
$-\odot$ AS-M+V
$*$ AS-H
$\rightarrow$ AS-H+V

(b)
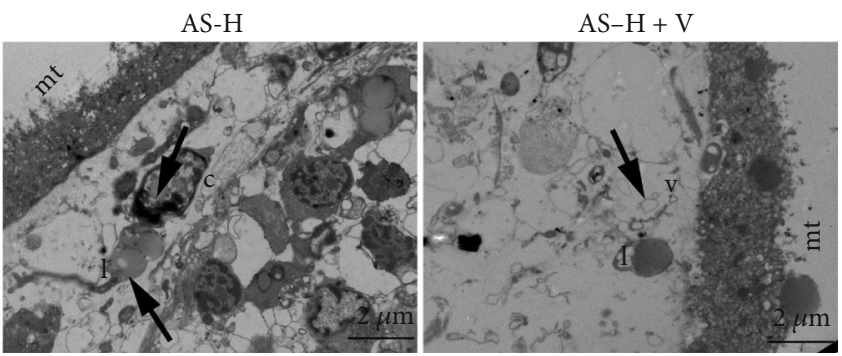

(c)

FIGURE 1: Viable rate of E. granulosus PSCs, activity of alkaline phosphatase in culture supernatant, and TEM analyses of drug-treated in vitro. PSCs were treated with DMSO, Veliparib $(10 \mu \mathrm{M})$, ABZ $(25 \mu \mathrm{M})$, AS-L $(65 \mu \mathrm{M})$, AS-M $(130 \mu \mathrm{M})$, AS-H $(325 \mu \mathrm{M})$, and AS-L/M/H+Veliparib $(10 \mu \mathrm{M})$. (a) Viability was determined through $1 \%$ eosin staining. (b) EgALP activity was measured in culture supernatants at different time points. (c) TEM analyses of E.g PSCs and DMSO group showed clear and visible cells, more and neat microtriches protruding from the tegument walls, and a normal germinal layer. mt: microtriches; c: parenchyma cell. Representative micrographs of protoscoleces after treatment with $\mathrm{ABZ}, \mathrm{AS}-\mathrm{L} / \mathrm{M} / \mathrm{H}$, and AS-L/M/H+Veliparib on day 4. l: lipid droplets; v: vacuoles; dc: appearance of small vacuoles in the distal cytoplasm; DMSO: dimethyl sulfoxide; V: Veliparib.

square test were used to analyze the comparison. $P<0.05$ was considered to be statistically significant.

\section{Results}

3.1. Effects of Drugs on the PSCs. PSCs were collected on days $1,2,3$, and 4 after treatment. On day 4 , the viability of PSCs in the DMSO group and Veliparib group was higher with a ratio of $100 \%$ and $94.27 \pm 1.19 \%$, respectively (Figure $1(\mathrm{a})$ ). The death rate of PSCs in the AS plus Veliparib group showed obvious increase compared with that of the single AS group $\left(\chi^{2}=104.155,24.748\right.$, and 34.016; $\left.P<0.01\right)$. This indicated that AS combined with Veliparib was superior to the AS group in killing the PSCs.

The activity of ALP in AS groups and the combination of AS and Veliparib group in PSCs showed a significant increase after treatment, which was in a time-dependent manner especially in the AS-H group and AS-H+Veliparib group (Figure 1(b)). On day 2, it reached the peak level, followed by a gradual decline. In the AS-H group or AS-H combined groups, the increase of ALP was the most obvious, which reached the peak level on day 3 in ABZ group. The activity of ALP in the combination of AS and Veliparib was more superior than that of the AS group.

The ultrastructural images of TEM are shown in Figure 1(c). DMSO and Veliparib-treated PSCs showed no ultrastructural alteration in PSCs tissue. There were many microvillus in the outer layer. The cell has a complete morphological structure, and the nuclear structure was dense. The villus in the outer layer in the ABZ group was no longer available, and the internal structure was not regular. The cellular structures were completely destroyed. AS-H treatment 
DMSO

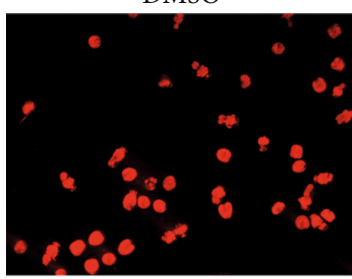

AS-L

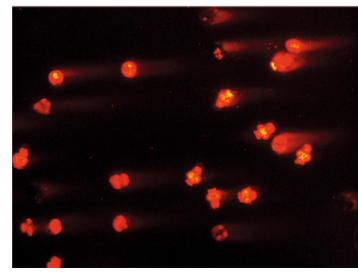

ABZ

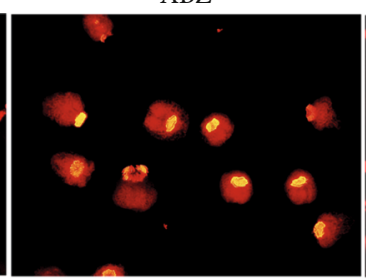

AS-H

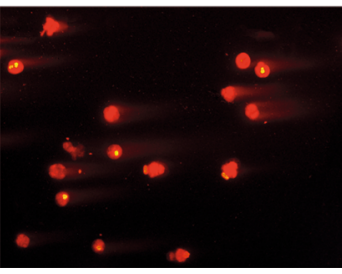

(a)

V

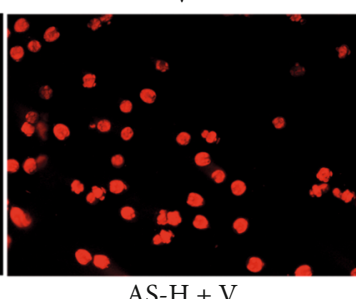
AS- $\mathrm{H}+\mathrm{V}$
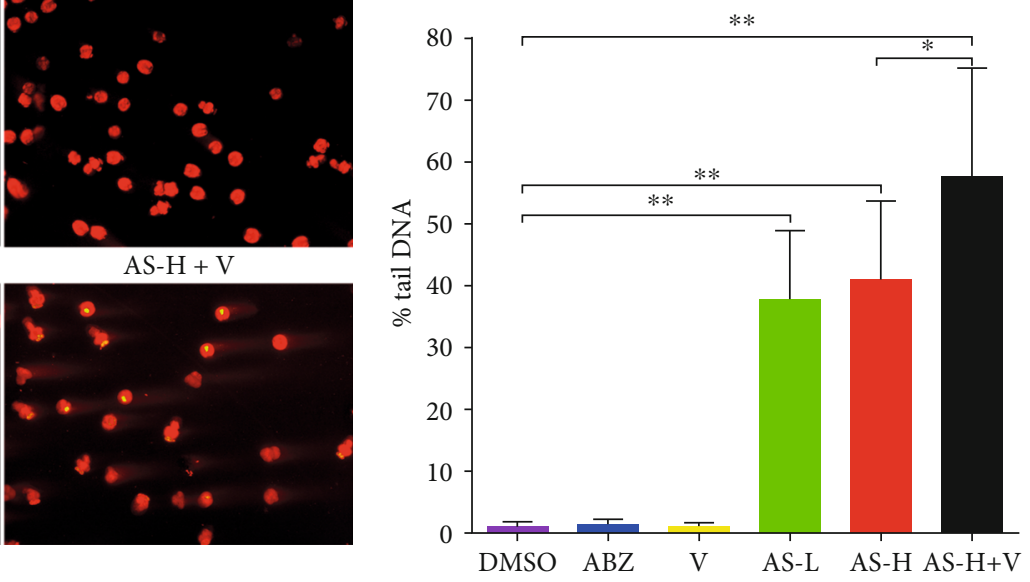

(b)

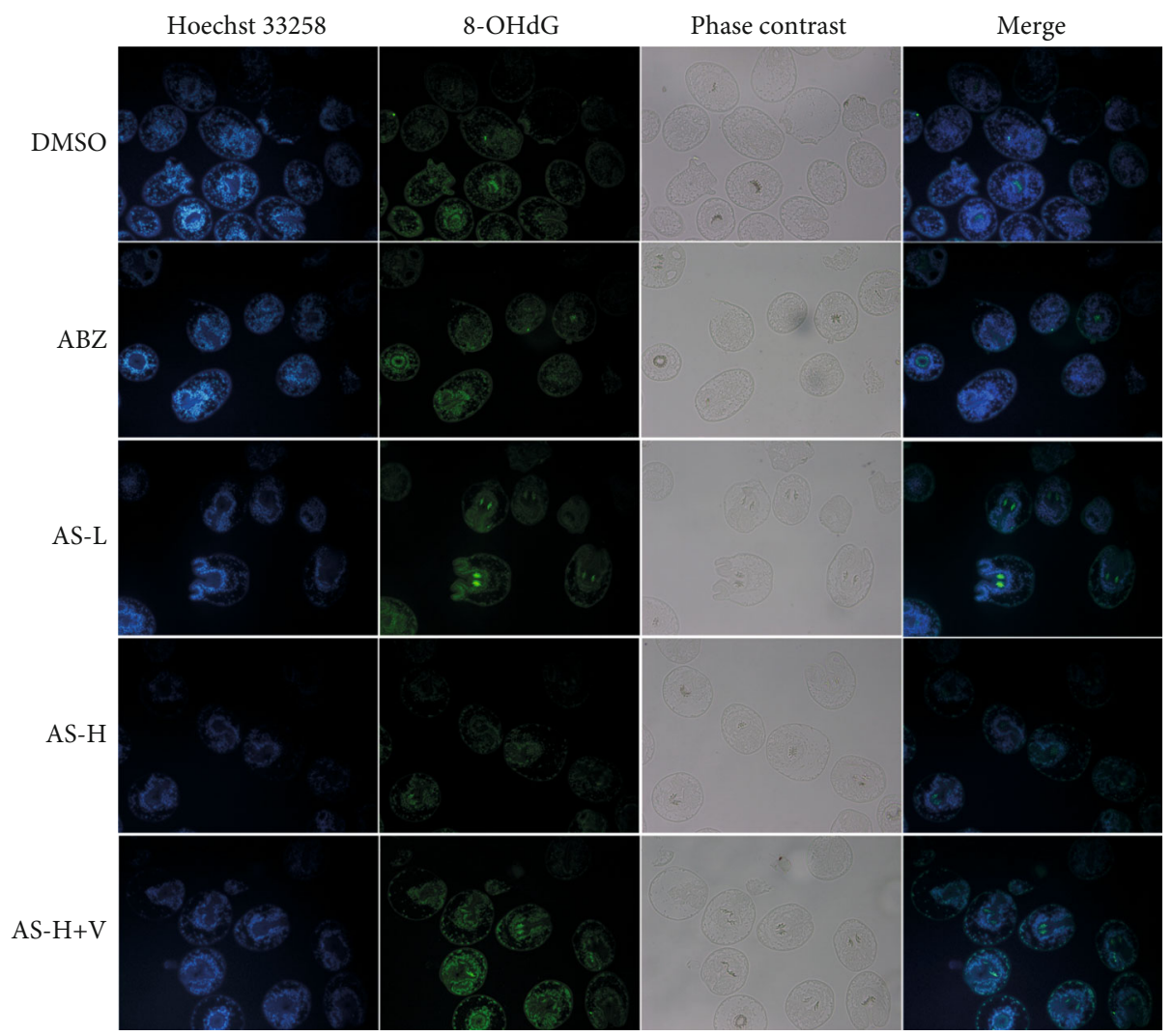

(c)

Figure 2: Continued. 


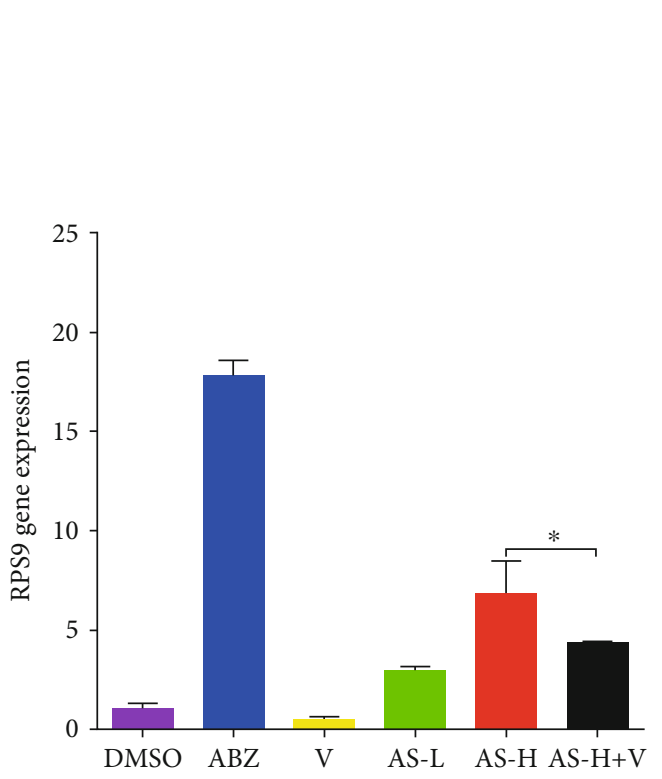

(d)

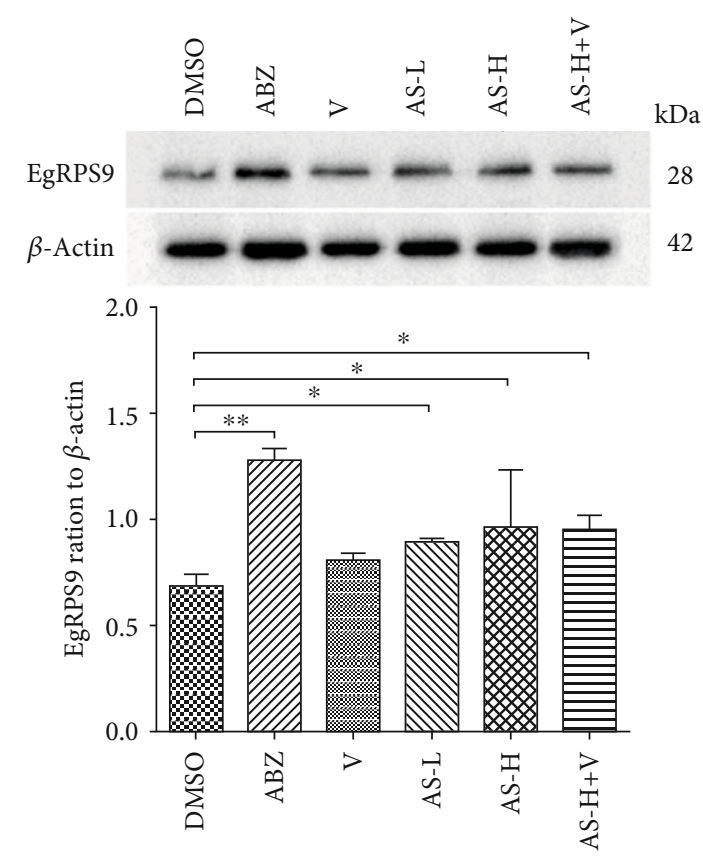

(e)

FIgURE 2: Correlation index detection in each group after the drug intervention. (a) The comet images of E.g PSCs on DNA damage. (b) Percentage tail DNA of comet value assay; ${ }^{*} P<0.05 ;{ }^{* *} P<0.01$. (c) Oxidative damage products of DNA in PSCs exposed to different concentrations of drugs in vitro, as determined by $8-\mathrm{OHdG}$ immunolocalization. PSCs were treated with DMSO, ABZ (25 $\mu \mathrm{M})$, AS-L $(65 \mu \mathrm{M})$, AS-H $(325 \mu \mathrm{M})$, and AS-H $(325 \mu \mathrm{M})+$ Veliparib $(10 \mu \mathrm{M})$ for 4 days. Hoechst was used for nuclear staining. Positive 8-OHdG nuclei, green color. (d) The gene expression levels of EgRPS9 in PSCs were evaluated by qRT-PCR and Western blot (e). DMSO: dimethyl sulfoxide; V: Veliparib.

showed disorder or even loss of microtriches, together with the presence of abnormalities in internal structure, lipid droplets, scattered nucleoli, and heterochromatin margination phenomenon. AS-H plus Veliparib showed an even more dramatic effect, which resulted in complete destruction of the PSCs tissues. The structural integrity of the germinal layer was totally lost, and the majority of the microtriches were absent. Besides, the distal cytoplasm was loose, together with the reduction of germinal layers. The lipid droplets and vacuoles were available. The results of ultrastructure observation were consistent with those of PSCs mortality and ALP activity.

3.2. Comet Assay in E.g PSCs. The DNA damages as revealed by comet assay are shown in Figure 2(a). The percentage of DNA in the tail value is shown in Figure 2(b). The morphology in the DMSO group, Veliparib group, and ABZ group was normal, with no tail formation. Compared with the Veliparib and DMSO groups, significant differences were noticed in the percentage of tail DNA values in the AS groups $(F=62.270, P<0.01)$. Compared with the AS-H group, differences were observed in the percentage of tail DNA values in the AS-H + Veliparib group $(t=2.491, P<0.05)$. Therefore, the tailing in the AS-H+Veliparib group showed higher DNA percentage in tail values, indicating the DNA damages were more severe in these groups.

3.3. Oxidative DNA Damage in E.g PSCs. The expression of 8 -OHdG in the PSCs of each group is shown in
Figure 2(c). There was a gradual increase in the number of positive nuclei for 8-OHdG (in green); upon treating with Veliparib, the expression of $8-\mathrm{OHdG}$ showed a significant increase compared with that of the AS-H group, indicative of oxidative DNA damage resulting from treatment with increased levels of AS and the combination of AS. Fluorescence was not detected in histological sections of PSCs treated with DMSO, Veliparib, and weak fluorescence with ABZ. PSCs presenting tegument damages (AS-H+Veliparib group) showed higher levels DNA damage in the AS-H+Veliparib group.

3.4. Analysis of EgRPS9 Expression in PSCs. The EgRPS9 expression level in the ABZ-treated group was 17.28-fold higher than the level in the DMSO group $(P<0.01$, Figure 2(d)). The EgRPS9 expression levels in the AS-Ltreated group and the AS-H treated group were 2.84-fold and 6.66-fold higher compared with that in the DMSO group, respectively $(P<0.05$, Figure $2(\mathrm{~d}))$. EgRPS9 expression level in the high AS-dose treated group was about 1.59-fold than that of the AS-H+Veliparib-treated group $(P<0.05)$.

3.5. EgRPS9 Identification by Western Blot. EgRPS9 protein of the drug-treated groups showed an increase compared with that of the DMSO group (Figure 2(e)), especially that of the ABZ group $(P<0.01)$. In the AS-H+Veliparib group, EgRPS9 was downregulated compared with that in the AS-H group despite no statistical difference $(P>0.05)$. 
3.6. AS and Veliparib Regulated the Growth Rate of Cysts in Infected Mice. Figure 3 shows the growth rate of the cystic volume. Within the 14 days, the growth rate in the AS groups showed a tendency of decline. The decline in the AS combined with the Veliparib group was more obvious than that in the other AS groups. Veliparib could enhance the activity of AS, which was presented by a maximal decline on day 15 in the AS-H+Veliparib group with the minimal growth rate.

$\mathrm{ABZ}, \mathrm{AS}-\mathrm{L} / \mathrm{H}$, and AS-H+Veliparib induced significant reduction in the cyst weights compared with the model group $(P<0.01$, Figure $4(\mathrm{a}))$. The mice treated with AS $(200 \mathrm{mg} / \mathrm{kg} / \mathrm{d})$ in combination with Veliparib exhibited reduction in cyst weight, which was higher than that of the AS group $(55.40 \%$ vs. $52.84 \%)$ and ABZ-treated mice (55.40\% vs. $55.35 \%)$. However, despite the improved mean efficacy of AS, there was no statistical difference in the cyst weight between the ABZ and AS alone or combinationtreated groups.

3.7. AS and Veliparib Induce Severe Ultrastructural Change on the Cystic Wall. Figure 4(b) indicates the ultrastructure of the cystic wall by TEM. The corneum layer and germinal layer of the cystic wall in the model group and Veliparib group were clear and normal. There was a large amount of microvillus, and the arrangement was regular. The nucleus was clearly displayed. In the ABZ group, the microvillus was no longer available, and the structure of the germinal layer was not clearly displayed. The amount of cells showed a decline, and the lipid droplet was observed. In AS-H group, the microvillus was no longer available, and the number of cells in the germinal layer showed decline, together with the presence of lipid droplet and vacuoles appeared. In the AS-H+Veliparib group, the microvillus was not available, and the structure of the germinal layer was porous and the texture was not regular. The cells were no longer available. Meanwhile, there were abundant lipid droplet and vacuoles.

3.8. Oxidative DNA Damage in Cysts Exposed to Different Concentrations of Drugs In Vivo. Figure 5 shows the representative Hoechst, 8-OHdG, and 8-OHdG/Hoechst merged images of the germinal layer (GL) from cysts. Generally, no positive 8 -OHdG reaction was observed in nuclei from the model group and ABZ group. The 8-OHdG positivity was observed in the GL of hydatid cysts treated with AS-H and AS-H+Veliparib, which represented the DNA damages. These results clearly suggest that oxidative damage of DNA was related to the drug intervention in E. granulosus hydatid cysts and PSCs.

\section{Discussion}

To our knowledge, this is the first report focusing on the effects of the combination of AS and Veliparib in cultured PSCs and the experimental Kunming mouse model. We determined the activity of PSCs after treatment using Eosin staining, which indicated that the PSCs activity decline was the most obvious in the AS-H+Veliparib group. In addition, we determined the activity of ALP in the PSCs, which indicated the activity showed significant elevation in those

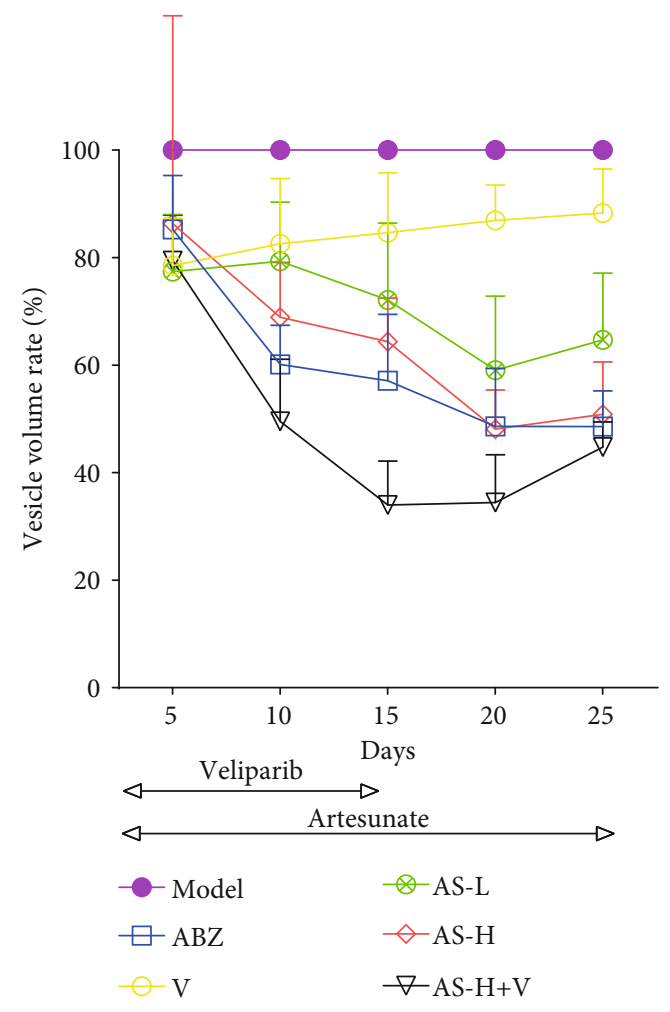

Figure 3: Changes of cyst volume in experimentally infected mice by ultrasonography. The vesicles growth rate was assessed by determining T/C \%. The model group received CMC-Na, and treatment groups were given Veliparib $(25 \mathrm{mg} / \mathrm{kg} / \mathrm{d}$, p.o., q.d.). On days $1-14$, AS-H $(200 \mathrm{mg} / \mathrm{kg} / \mathrm{d})$ was administered $2 \mathrm{~h}$ after Veliparib. On days 1-30, ABZ (200 mg/kg/d), AS-L (50 mg/kg/d), and AS-H (200 mg/kg/d, p.o., q.d.) were administered.

with severe injury. Our data showed that the activity of ALP showed an obvious increase in the AS-H+Veliparib group. On day 2 , it reached the peak level, followed by a gradual decline, which may be related to the increase of PSCs death after treatment resulting in the decline in the release of enzymes.

In the in vivo experiments, mice were infected with $\mathrm{CE}$ and received AS-H and Veliparib on days 1-14, followed by AS administration via lavage after 14 days. Ultrasonography was performed to monitor the cyst volume per week during the 6 weeks. The cystic growth rate in the AS-H+Veliparib group showed a significant decline within 14 days. Compared with the AS-H group, the cyst volume in the AS-H+Veliparib group reached the lowest level at 15 days $(P<0.05)$, followed by a gradual increase of the cystic volume. Within 6 weeks after administration, the volume size in the ABZ group showed a gradual decline and reached a level of $48.57 \pm$ $6.63 \%$ on day 25 . After drug termination, the wet weight of cyst in the ABZ group and AS-H+Veliparib group showed a significant decline compared with the model group $(P<0.01)$, especially the ABZ group. No statistical differences were noticed between the ABZ group and AS-H+Veliparib group.

In the presence of oxygen, oxidation metabolism of PSCs or the immune response of the host was derived from ROS 


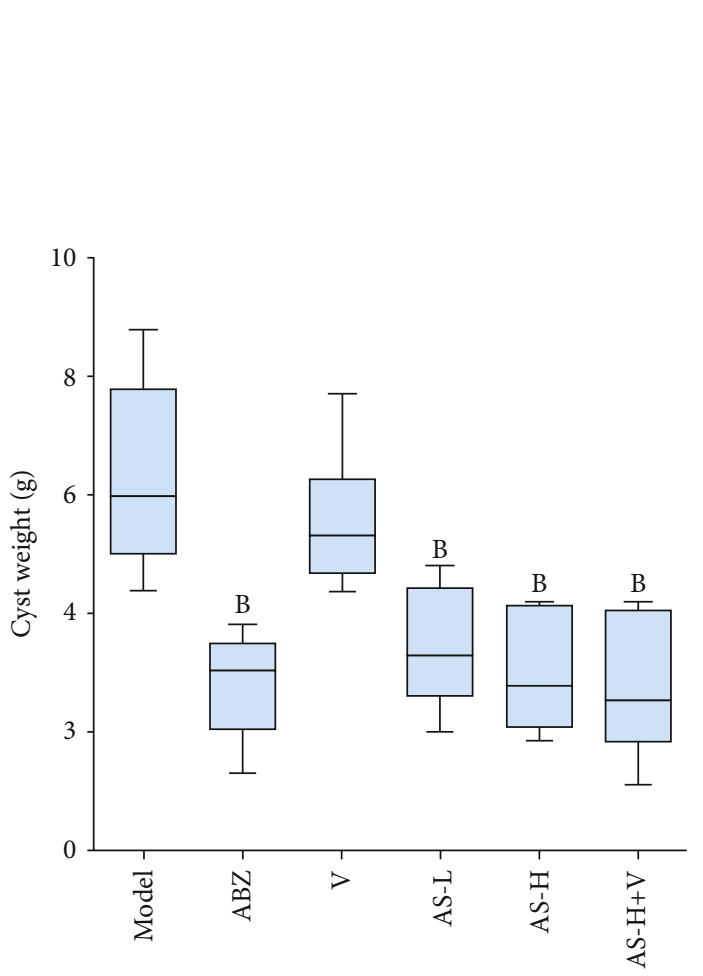

(a)

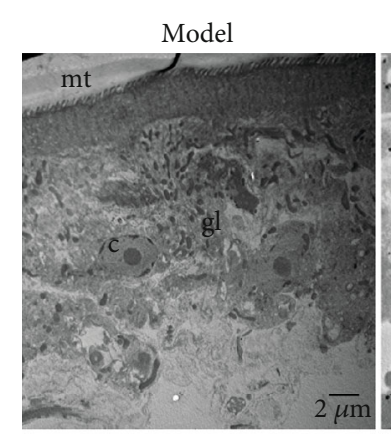

AS-H
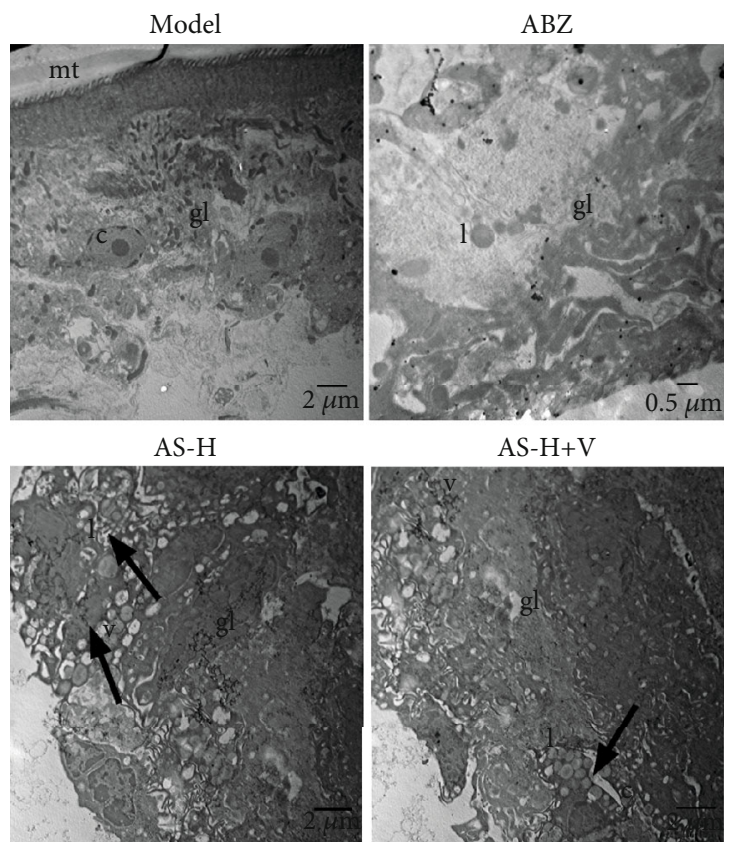

AS- $\mathrm{H}+\mathrm{V}$

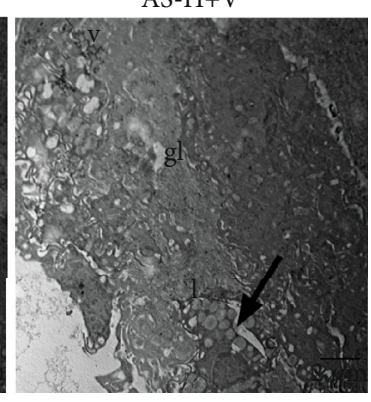

(b)

FIGURE 4: In vivo efficacy response of Veliparib in combination with AS in cystic echinococcosis models. (a) After euthanasia, the cysts were resected and weighed. Data consists of 10 mice per treatment group; bars, SE. For statistical analysis and being compared with the model group, ${ }^{\mathrm{B}} \mathrm{P}<0.01$. (b) TEM analyses of the cystic wall after in vivo treatment with ABZ, AS-H, and AS-H+Veliparib. The CMC-treated cystic walls showed that the germinal layer (gl) and clearly visible cells were undifferentiated cells (c) and the microtriches (mt) protruded from the tegument wall to laminated layer. Representative micrographs of the cystic wall collected from the infected Kunming mice after the daily lavage of ABZ $(200 \mathrm{mg} / \mathrm{kg} / \mathrm{d})$, AS-H $(200 \mathrm{mg} / \mathrm{kg} / \mathrm{d})$, and AS-H $(200 \mathrm{mg} / \mathrm{kg} / \mathrm{d})+$ Veliparib (25 mg/kg/d). l: lipid droplets; v: vacuoles; V: Veliparib.

and reactive nitrogen species [31,32]. To assess the potential effects of AS on DNA damages, comet assay based on single-cell electrophoresis was performed. Such method quantified the DNA damage as the percentage of DNA in tail. Our data showed that AS induced DNA damages in a dose-dependent manner. This implied that the ROS released by AS surpassed the natural oxygen consumption which led to oxidative stress triggering DNA damages in vivo. Indeed, base-excision repair was reported in the Schistosoma mansoni [33]. Wen et al. [18] reported a kinship between PSCs and S. mansoni based on the EgRPS9 gene analysis. Therefore, the BER mechanism may exist in PSCs. On this basis, we utilized the combination of AS and Veliparib, which indicated that Veliparib could induce more severe DNA damages.

In our previous study, we firstly cloned the RPS9 gene from the PSCs of E. granulosus after AS treatment [18]. It has been well acknowledged that RPS9 gene was closely involved in the various developmental stages of the E.g PSCs, such as DNA repair [34]. Therefore, we tried to investigate the expression of RPS9 gene after Veliparib treatment. Real-time PCR indicated that the expression of RPS9 was upregulated in the presence of AS, in a dose-dependent manner. After treating with the combination of AS and Veliparib, the expression of RPS9 gene showed downregu- lation compared with the AS-H group $(P<0.05)$. Western blot analysis showed that, after AS-L/H interference, the expression of RPS 9 protein was significantly higher than that of the DMSO group $(P<0.05)$. The combination of AS-H+Veliparib led to downregulation of RPS9 compared to that of the AS-H group. In the future, further studies are required to investigate the potential mechanisms of the combination of AS and Veliparib in downregulating the expression of RPS9 gene. According to the previous description, the activity of PARP-1 was relied on P53 as P53 could modulate the activity of RARP-1 through acetylation of SIRT1 [35]. RPS9 deficiency may activate P53, which then resulted in the apoptosis and aging process of cancer cells. Therefore, PARP-1 may be associated with the expression of RPS9 gene. Meanwhile, the expression of RPS9 gene was obviously upregulated in the ABZ group. To our best knowledge, $\mathrm{ABZ}$ could block the nutrition and glucose ingestion in the PSCs through modulating the metabolism of sulfoxide, sulfone, and 2-amine sulfone, which then resulted in glycogen depletion, together with the inhibition of the fumarate reductase. Subsequently, the generation of ATP was blocked, followed by survival and proliferation inhibition. These indicated that the pesticidal effects of ABZ may be associated with the expression of RPS9 gene and/or protein. On this basis, more studies are required to further illustrate this. 


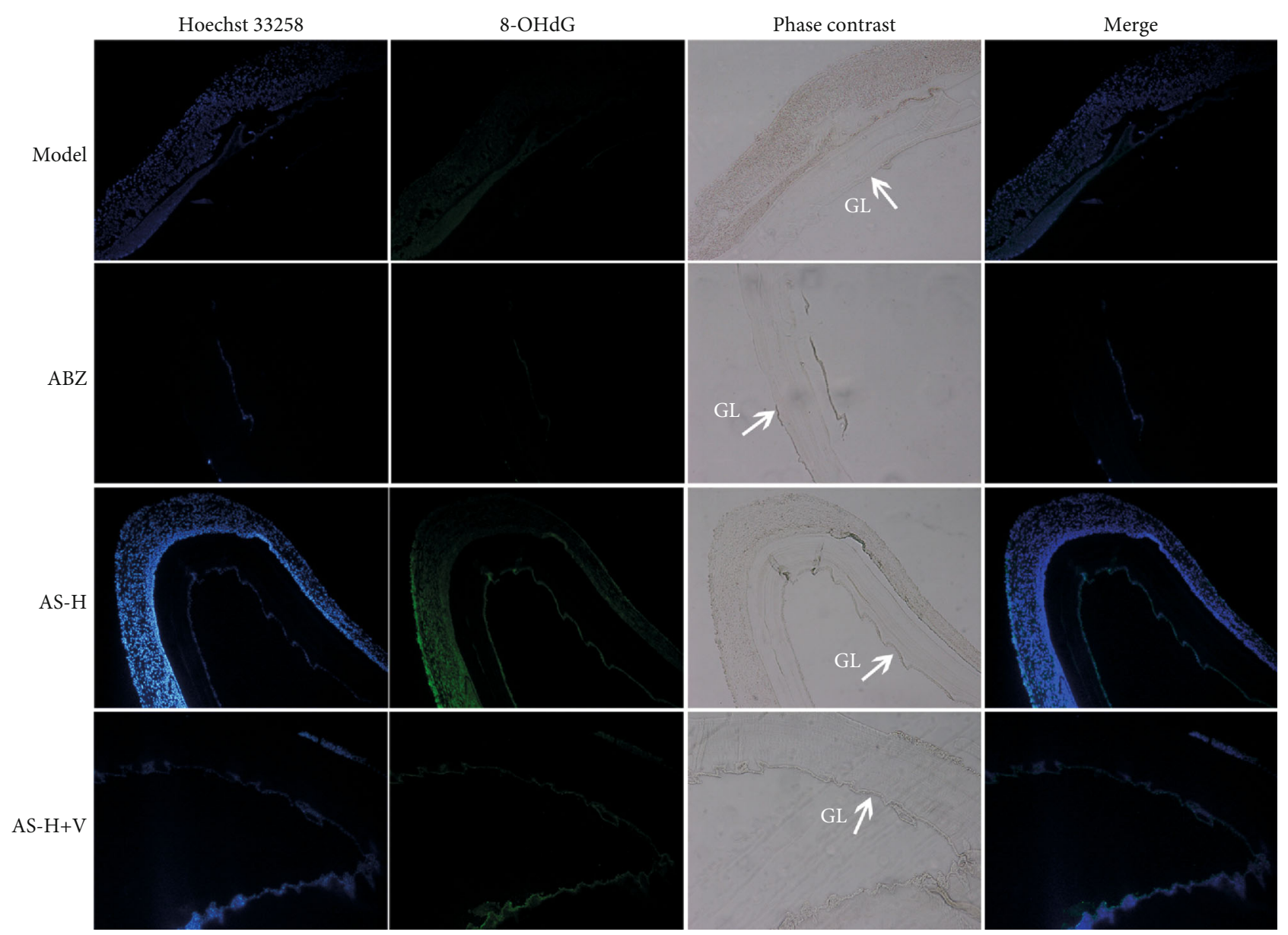

FIGURE 5: Oxidative DNA damage in cysts exposed to different concentrations of drugs in vivo, as determined by 8-OHdG immunofluorescence. The mice were treated with ABZ (200 mg/kg/d), AS-H (200 mg/kg/d), and AS-H+Veliparib (25 mg/kg/d). Hoechst was used for nuclear staining. Parts showing positive 8-OHdG nuclei (green). V: Veliparib.

For the limitations of this study, we cannot confirm which molecule is involved in the antiparasitic effects of AS on CE. In this study, we preliminarily investigated the molecular mechanism of AS on the CE. In the future, further studies are required to fully illustrate the exact mechanism.

In summary, we firstly reported that Veliparib could enhance the antiparasitic effects of AS on CE. Our data showed that the efficiency of AS on CE may be associated with inducing DNA damages, which then played an important role in the antihydatid cyst diseases. Our study paved the way for the research and development of drugs based on AS for treating CE.

\section{Data Availability}

All the data were available upon appropriate request.

\section{Conflicts of Interest}

The authors declare that there is no conflict of interest regarding the publication of this paper.

\section{Acknowledgments}

This work was supported by the National Natural Science Foundation of China (nos. 81560607 and 81860666) and State Key Laboratory of Pathogenesis, Prevention, Treatment of High Incidence Diseases in Central Asia Fund (no. SKLHIDCA-2018-5).

\section{References}

[1] P. E. Pensel, M. A. Maggiore, L. B. Gende, M. J. Eguaras, M. G. Denegri, and M. C. Elissondo, "Efficacy of essential oils of Thymus vulgaris and Origanum vulgare on Echinococcus granulosus," Interdisciplinary perspectives on infectious diseases, vol. 2014, Article ID 693289, 12 pages, 2014.

[2] M. Rajabloo, S. H. Hosseini, and F. Jalousian, "Morphological and molecular characterisation of Echinococcus granulosus from goat isolates in Iran," Acta Tropica, vol. 123, no. 2, pp. 67-71, 2012.

[3] E. Brunetti, P. Kern, and D. A. Vuitton, "Expert consensus for the diagnosis and treatment of cystic and alveolar echinococcosis in humans," Acta Tropica, vol. 114, no. 1, pp. 1-16, 2010.

[4] G. L. Amidon, H. Lennernas, V. P. Shah, and J. R. Crison, "A theoretical basis for a biopharmaceutic drug classification: the correlation of in vitro drug product dissolution and 
in vivo bioavailability," Pharmaceutical research, vol. 12, no. 3, pp. 413-420, 1995.

[5] K. Daniel-Mwambete, S. Torrado, C. Cuesta-Bandera, F. Ponce-Gordo, and J. J. Torrado, "The effect of solubilization on the oral bioavailability of three benzimidazole carbamate drugs," International journal of pharmaceutics, vol. 272, no. 1-2, pp. 29-36, 2004.

[6] B. E. Gama, N. de Oliveira, J. M. de Souza et al., "Brazilian Plasmodium falciparum isolates: investigation of candidate polymorphisms for artemisinin resistance before introduction of artemisinin-based combination therapy," Malaria Journal, vol. 9, no. 1, p. 355, 2010.

[7] L. Jones-Brando, J. D'Angelo, G. H. Posner, and R. Yolken, "In vitro inhibition of Toxoplasma gondii by four new derivatives of artemisinin," Antimicrobial Agents and Chemotherapy, vol. 50, no. 12, pp. 4206-4208, 2006.

[8] A. C. Cala, J. F. S. Ferreira, A. C. S. Chagas et al., "Anthelmintic activity of Artemisia annua L. extracts in vitro and the effect of an aqueous extract and artemisinin in sheep naturally infected with gastrointestinal nematodes," Parasitology Research, vol. 113, no. 6, pp. 2345-2353, 2014.

[9] T. K. Våtsveen, M. R. Myhre, C. B. Steen et al., "Artesunate shows potent anti-tumor activity in B-cell lymphoma," Journal of Hematology \& Oncology, vol. 11, no. 1, p. 23, 2018.

[10] M. Spicher, A. Naguleswaran, L. M. Ortega-Mora, J. Muller, B. Gottstein, and A. Hemphill, "In vitro and in vivo effects of 2-methoxyestradiol, either alone or combined with albendazole, against Echinococcus metacestodes," Experimental Parasitology, vol. 119, no. 4, pp. 475-482, 2008.

[11] M. Stettler, J. F. Rossignol, R. Fink et al., "Secondary and primary murine alveolar echinococcosis: combined albendazole/nitazoxanide chemotherapy exhibits profound anti-parasitic activity," International Journal for parasitology, vol. 34, no. 5, pp. 615-624, 2004.

[12] M. Spicher, C. Roethlisberger, C. Lany et al., "In vitro and in vivo treatments of echinococcus protoscoleces and metacestodes with artemisinin and artemisinin derivatives," Antimicrobial agents and chemotherapy, vol. 52, no. 9, pp. 34473450, 2008.

[13] O. Natalang, E. Bischoff, G. Deplaine et al., "Dynamic RNA profiling in Plasmodium falciparum synchronized blood stages exposed to lethal doses of artesunate," BMC Genomics, vol. 9, no. 1, p. 388, 2008.

[14] T. Antoine, N. Fisher, R. Amewu, P. M. O'Neill, S. A. Ward, and G. A. Biagini, "Rapid kill of malaria parasites by artemisinin and semi-synthetic endoperoxides involves ROSdependent depolarization of the membrane potential," Journal of Antimicrobial Chemotherapy, vol. 69, no. 4, pp. 1005-1016, 2014.

[15] C. L. Hartwig, A. S. Rosenthal, J. D'Angelo, C. E. Griffin, G. H. Posner, and R. A. Cooper, "Accumulation of artemisinin trioxane derivatives within neutral lipids of Plasmodium falciparum malaria parasites is endoperoxide-dependent," Biochemical Pharmacology, vol. 77, no. 3, pp. 322-336, 2009.

[16] N. J. White, "Qinghaosu (artemisinin): the price of success," Science, vol. 320, no. 5874, pp. 330-334, 2008.

[17] G. Lü, W. Zhang, J. Wang et al., "Application of a cDNA microarray for profiling the gene expression of Echinococcus granulosus protoscoleces treated with albendazole and artemisinin," Molecular and Biochemical Parasitology, vol. 198, no. 2, pp. 59-65, 2014.
[18] L. M. Wen, G. D. Lü, J. Zhao et al., "Molecular cloning and characterization of ribosomal protein RPS9 inEchinococcus granulosus," Journal of Parasitology, vol. 103, no. 6, pp. 699707, 2017.

[19] Y. Cong, L. Wang, Z. Wang et al., "Enhancing therapeutic efficacy of cisplatin by blocking DNA damage repair," ACS Medicinal Chemistry Letters, vol. 7, no. 10, pp. 924-928, 2016.

[20] S. Madhusudan and M. R. Middleton, "The emerging role of DNA repair proteins as predictive, prognostic and therapeutic targets in cancer," Cancer Treatment Reviews, vol. 31, no. 8, pp. 603-617, 2005.

[21] Y. Luo, X. Ji, F. Ling et al., "Impaired DNA repair via the baseexcision repair pathway after focal ischemic brain injury: a protein phosphorylation-dependent mechanism reversed by hypothermic neuroprotection," Frontiers in Bioscience, vol. 12, no. 1, pp. 1852-1862, 2007.

[22] R. Wang, C. Li, P. Qiao et al., "OGG1-initiated base excision repair exacerbates oxidative stress-induced parthanatos," Cell death \& disease, vol. 9, no. 6, p. 628, 2018.

[23] D. Fu, J. A. Calvo, and L. D. Samson, "Balancing repair and tolerance of DNA damage caused by alkylating agents," Nature Reviews Cancer, vol. 12, no. 2, pp. 104-120, 2012.

[24] T. K. Owonikoko, G. Zhang, X. Deng et al., "Poly (ADP) ribose polymerase enzyme inhibitor, veliparib, potentiates chemotherapy and radiation in vitro and in vivo in small cell lung cancer," Cancer medicine, vol. 3, no. 6, pp. 1579-1594, 2014.

[25] M. Stettler, M. Siles-Lucas, E. Sarciron, P. Lawton, B. Gottstein, and A. Hemphill, "Echinococcus multilocularis alkaline phosphatase as a marker for metacestode damage induced by in vitro drug treatment with albendazole sulfoxide and albendazole sulfone," Antimicrobial agents and chemotherapy, vol. 45, no. 8, pp. 2256-2262, 2001.

[26] M. Antoniou and Y. Tselentis, "Studies on Echinococcus granulosus using the scanning electron microscope. I. Preparations of the parasite for infection of the final host," Parasitology research, vol. 79, no. 7, pp. 537-542, 1993.

[27] N. P. Singh, M. T. McCoy, R. R. Tice, and E. L. Schneider, "A simple technique for quantitation of low levels of DNA damage in individual cells," Experimental cell research, vol. 175, no. 1, pp. 184-191, 1988.

[28] A. R. Collins, "The comet assay for DNA damage and repair: principles, applications, and limitations," Molecular biotechnology, vol. 26, no. 3, pp. 249-261, 2004.

[29] G. Cabrera, M. E. Cabrejos, A. L. Morassutti et al., "DNA damage, RAD9 and fertility/infertility ofEchinococcus granulosus hydatid cysts," Journal of cellular physiology, vol. 216, no. 2, pp. 498-506, 2008.

[30] W. B. Zhang, M. K. Jones, J. Li, and D. P. McManus, "Echinococcus granulosus: pre-culture of protoscoleces in vitro significantly increases development and viability of secondary hydatid cysts in mice," Experimental parasitology, vol. 110, no. 1, pp. 88-90, 2008.

[31] M. Conchedda, E. Gabriele, and G. Bortoletti, "Immunobiology of cystic echinococcosis," Parassitologia, vol. 46, no. 4, pp. 375-380, 2004.

[32] H. L. Callahan, R. K. Crouch, and E. R. James, "Helminth anti-oxidant enzymes: a protective mechanism against host oxidants?," Parasitology Today, vol. 4, no. 8, pp. 218-225, 1988.

[33] C. Furtado, C. G. Regis-da-Silva, D. G. Passos-Silva et al., "Schistosoma mansoni: the IMP4 gene is involved in DNA 
repair/tolerance after treatment with alkylating agent methyl methane sulfonate," Experimental parasitology, vol. 116, no. 1, pp. 25-34, 2007.

[34] L. Pnueli and Y. Arava, "Genome-wide polysomal analysis of a yeast strain with mutated ribosomal protein S9," BMC Genomics, vol. 8, no. 1, p. 285, 2007.

[35] J. Montero, C. Dutta, D. van Bodegom, D. Weinstock, and A. Letai, "p53 regulates a non-apoptotic death induced by ROS," Cell Death \& Differentiation, vol. 20, no. 11, pp. 14651474, 2013. 